\title{
COSTRUTTIVISMO E REALISMO NELLE SCIENZE FISICHE
}

\author{
PAOLO PERINOTTI (*)
}

SunTO. - Mentre la fisica classica ben si concilia con il punto di vista oggettivista, l'avvento della fisica contemporanea ne mette in discussione gli assunti fondamentali. Il dilemma che la meccanica quantistica impone alla filosofia della scienza è se l'interpretazione intrinsecamente probabilistica della meccanica quantistica debba essere rivisitata, per salvare il tradizionale impianto epistemologico, oppure debba essere adottata insieme alle sue conseguenze. Si propongono i principali argomenti in favore della seconda opzione. Si discute inoltre di come tale scelta possa suggerire una visione costruttivista delle scienze fisiche.

$* * *$

ABSTRACT. - While classical physics is consistent with an objectivist point of view, the advent of modern physics undermines its fundamental assumptions. Quantum mechanics, in particular, put a question to the philosophy of science as to whether its intrinsically probabilistic interpretation should be revised in order to save the traditional epistemology, or should be adopted unconditionally, along with its revolutionary consequences. We propose some crucial arguments in favour of the second option. Finally, we discuss how the above choice may suggest a constructivist view about the nature of physical sciences.

Quale valore gnoseologico dobbiamo attribuire ad una teoria scientifica? In particolare quale valore ha una teoria fisica? Ovvero, quale tipo di conoscenza possiamo acquisire dalla scienza fisica? Lo scopo del presente contributo è quello di fornire uno spunto per affrontare questa questione.

In primo luogo, è opportuno chiarire in modo definitivo che cosa

(*) Dipartimento di Fisica, Università degli Studi di Pavia, Italia.

E-mail: paolo.perinotti@unipv.it 
si intenderà come teoria fisica nel seguito, in modo da liberare il campo da equivoci su tale punto fondamentale. Per "teoria fisica" intenderemo una teoria matematica, affiancata da una mappa che associ agli oggetti matematici determinati enti fisici. In particolare, gli enti fisici saranno individuati in termini di protocolli, sequenze di istruzioni, esprimibili in qualunque linguaggio, senza fare ricorso al linguaggio matematico della teoria stessa. ${ }^{1}$

A titolo di esempio, consideriamo la meccanica razionale Lagrangiana: in essa si fa riferimento a concetti, quali quelli di punto materiale e di corpo rigido, che sono entità puramente matematiche. Ciò che fa della meccanica Lagrangiana una teoria fisica è la possibilità di descrivere un determinato sistema concreto come punto materiale o come corpo rigido. A tal fine, la forma, la massa, la posizione, la velocità del corpo, che sono variabili matematiche nella teoria, corrisponderanno ai risultati di misure effettuate sull'oggetto modellizzato come punto materiale o come corpo rigido, mediante determinate procedure sperimentali. Sono queste ultime ad individuare gli enti fisici, quali forma, massa, posizione, velocità, e via dicendo. Si noti che possiamo descrivere la procedura per misurare la posizione di un sistema senza far riferimento alcuno alla sua modellizzazione astratta come ente matematico.

È chiaro come, adottando questo punto di vista sulla teoria fisica, il suo status gnoseologico sia determinato da due fattori, non completamente indipendenti:

1. Il valore che attribuiamo alla matematica;

2. Il valore che attribuiamo alla relazione tra oggetti matematici e oggetti fisici.

Per quanto riguarda il primo punto, le principali posizioni che mi propongo di considerare sono due: la prospettiva Platonista/realista e quella costitutivista/concettualista. ${ }^{2}$

1 Tale definizione, che giace alla base di alcuni approcci operazionali alla meccanica quantistica, nasce dall'istanza pragmatica di separare concettualmente gli enti teorici da quelli concreti, ovvero dai dati sensoriali - prima ancora che sperimentali. A conoscenza di chi scrive essa è da attribuire, con beneficio di opportuna parafrasi, a Günther Ludwig [1].

2 Per una dettagliata discussione di tali prospettive si veda ad esempio [2]. 
Riassumiamo per completezza tali posizioni in estrema sintesi. L'approccio Platonista attribuisce agli enti matematici una esistenza indipendente dalla possibilità del linguaggio di esprimerli e caratterizzarli. Al contrario, la posizione costitutivista vede nella definizione matematica l'atto costitutivo di un ente altrimenti non esistente. In tal modo l'intera matematica risulta in primis come una costruzione dell'intelletto umano.

In questa dicotomia, una posizione realista nei confronti delle teorie fisiche è conciliabile solamente con una posizione Platonista riguardo alla matematica. Infatti, se la teoria matematica che costituisce l'ossatura teorica della fisica è una mera costruzione dell'intelletto umano, allora il proposito della scienza fisica non può essere quello di spiegare la realtà così come essa è, avendo a disposizione un linguaggio limitato. In tal caso, il ruolo della scienza fisica diventa quello di costruire un linguaggio con adeguate regole, che serva a parlare in modo accurato degli eventi fisici, dandone una descrizione consistente e permettendone predizioni affidabili. Ė da notare che, al contrario, quest'ultima visione del ruolo della fisica è anche compatibile con una visione Platonista/realista dei concetti matematici, concepiti quindi come reali indipendentemente dal loro legame con i dati empirici. Difficilmente lo sviluppo della fisica potrà fornire indizi in favore di una scelta o dell'altra.

Sulla seconda questione, invece, riteniamo che la fisica possa fornire utili indicazioni. Concentreremo quindi nel seguito l'attenzione sul secondo punto, ovvero il legame tra enti teorici ed enti fisici, prendendo ispirazione dalla meccanica quantistica.

I concetti introdotti per rendere conto dei nuovi fenomeni che interrogavano i fisici di fine Ottocento non hanno prodotto tanto un progresso della conoscenza fisica, quanto una vera e propria palingenesi. Tale rivoluzione ha inevitabilmente coinvolto il modo di concepire la realtà. La meccanica quantistica ha trovato una formulazione consistente e soddisfacente solo in seguito alla proposta di Max Born, di interpretare le "funzioni d'onda" come ampiezze di probabilità. Nacque allora una domanda che agita ancora oggi profondamente la riflessione sulla meccanica quantistica. Come può una teoria che ambisca a descrivere i fenomeni elementari del mondo fisico essere probabilistica? E ancora: come può una teoria fondamentale fare riferimento ad un concetto come la misurazione?

Ad una attenta analisi, appare evidente che tali domande celino una assunzione: ovvero che la totalità degli eventi osservati e delle loro relazioni siano intelligibili ad una teoria fisica. Questa assunzione è precisa- 
mente il nodo che mi propongo di affrontare, ed è a questo punto che entra in gioco la proposta suggerita nel titolo.

Per quanto il costruttivismo sia una corrente più genuinamente collocata nel contesto della meta-etica e delle scienze cognitive, ${ }^{3}$ essa ben descrive la visione che si desidera qui sostenere a proposito del ruolo gnoseologico di una teoria scientifica alla luce della fisica contemporanea.

In breve, la dottrina del costruttivismo sostiene che la conoscenza non si riduca all'acquisizione immediata da parte dell'intelletto della realtà così come essa oggettivamente è, ma consista in una paziente architettura della mente umana, sempre in crescita e rielaborazione, e sempre più efficace nell'informare le interazioni con l'ambiente, senza necessariamente riferirsi a verità oggettive. Non è pertanto centrale, in tale approccio alla conoscenza, il legame della teoria con una postulata realtà indipendente dall'osservatore, quanto invece la sua capacità di predire ed improntare l'interazione con l'ambiente fisico. Ci proponiamo nel seguito di argomentare in favore di tale posizione, concentrando la riflessione su alcuni specifici aspetti della teoria quantistica.

Gli eventi che la teoria quantistica permette di prevedere sono gli esiti di misure condotte su sistemi fisici preparati e trasformati con determinate procedure. Supponiamo di rappresentare una preparazione del sistema designato con A (ad esempio, lo spin di un elettrone accelerato in un sincrotrone) mediante un diagramma come il seguente:

\section{$\rho \mathrm{A}$.}

Ora, l'atto della misura di una grandezza del sistema consiste in una trasformazione che avviene contestualmente alla produzione del risultato, poniamo che esso sia " $i$ ". Ad esempio, misurando la componente lungo l'asse verticale $z$ dello spin dell'elettrone accelerato ed emesso dal sincrotrone, si può ottenere " $i=1 / 2$ " oppure " $i=-1 / 2$ ". In tal caso la trasformazione subita dallo spin costituisce la preparazione di un nuovo stato. Schematizzeremo tale evento con il seguente diagramma:

\section{$\rho \wedge \mathscr{A}_{i} \mathrm{~A}$.}

Per un sistema quantistico, come lo spin dell'elettrone, non c'è modo di ricavare un'informazione sul sistema senza produrre su di esso una trasformazione non banale. Tale principio è noto come "no information without disturbance" [3]. Esso ha conseguenze dirompenti proprio 
sul punto che ci si propone di analizzare in questa sede, ovvero il legame tra descrizione teorica di un processo fisico ed il processo stesso. Infatti, mentre le leggi postulate dalla fisica riguardo sistemi isolati, e ad oggi ampiamente corroborate dalle osservazioni, sono reversibili, il processo di misurazione è irreversibile.

Tuttavia, il processo di misurazione non riguarda sistemi isolati, bensì sistemi aperti, in interazione con l'osservatore. Le trasformazioni dei sistemi quantistici aperti hanno una peculiarità: dal punto di vista matematico esse si possono pensare come interazioni reversibili con un secondo sistema $\mathrm{E}$, il quale, in seguito all'interazione, subisce a sua volta una misura, e viene scartato. Lo schema è il seguente:

$$
\rho \mathrm{A} \mathscr{A}_{i} \mathrm{~A}=\rho_{\eta}^{\rho \mathrm{E}} \mathscr{U}_{\mathrm{E}}^{\mathrm{A}} P_{i} .
$$

Questa osservazione fornisce una illusoria via di uscita dalla necessità di introdurre il concetto di "misurazione" a livello fondamentale. Alla luce di tale osservazione, infatti, è immediato immaginare che si possa estendere il sistema fisico in considerazione ad arbitrio. In un celebre esempio, von Neumann descrive questa idea con le parole seguenti: “. . . it is rather arbitrary whether or not one includes the observer in $\mathrm{M}$ [il sistema $\mathrm{E}$ nel nostro caso], and replaces the relation between the $\mathrm{S}$ state [stato del sistema $\mathrm{A}$ ] and the pointer positions in $\mathrm{M}$ by the relations of this state and the chemical changes in the observer's eye, or even in his brain." [4]. Dal punto di vista diagrammatico, tale procedimento è illustrato spostando il sottosistema sottoposto a "misurazione" sempre più remotamente, come nel diagramma seguente.

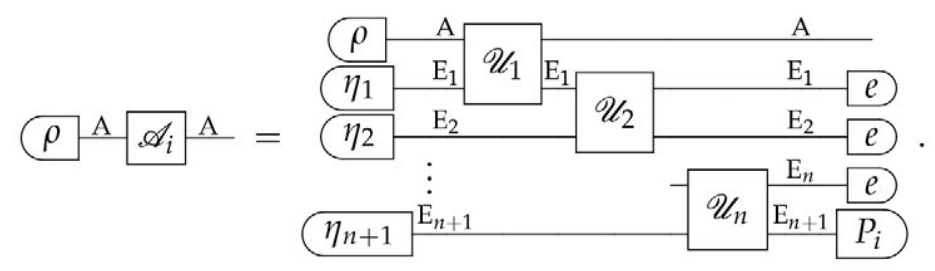

Gli eventi indicati con il simbolo " $e$ " rappresentano semplicemente il fatto che il sistema su cui essi agiscono è ignorato nelle successive fasi del processo di misurazione.

Tuttavia, per quanto remotamente si sposti l'evento irreversibile di misurazione, per avere l'equivalenza con il diagramma originale, esso 
non può sparire: deve prima o poi essere introdotto, e l'unica scelta che si ha è quanto dettaglio dell'apparato di misurazione introdurre nella descrizione teorica del processo. D'altro canto, se si estende lo schema indefinitamente, sino ad includere tutti i sistemi che costituiscono l'universo, e si rimuove l'evento di misurazione, il modello matematico che si ottiene non sarà in grado di rendere conto della formazione di un risultato della misura e di calcolarne le probabilità. Quest'ultima situazione è quella prefigurata dall'interpretazione "many-worlds", che trova tuttavia non poche difficoltà, in primis la necessità di giustificare le predizioni probabilistiche della meccanica quantistica.

La teoria quantistica, per poter essere una teoria fisica nel senso indicato all'inizio del contributo, necessita quindi del concetto di misurazione, non essendo altrimenti confrontabile con i dati dell'esperienza. Va detto che si può obiettare, ovviamente, a tale posizione in modo radicale, sostenendo, come talvolta avviene da parte dei fautori dell'interpretazione "many-worlds", che gli eventi che ci appaiono sono accidentali e contingenti, e non hanno quindi la rilevanza ad essi attribuita in prima istanza dalla nostra suddivisione della teoria fisica in una teoria matematica ed in una mappa che associ agli oggetti matematici determinati enti fisici. In particolare, per effetto di tale obiezione, il secondo elemento, che connette l'apparato teorico alle procedure sperimentali, perde di importanza.

Tuttavia, in tale prospettiva, diventa difficile sostenere come dall'esperienza di eventi fisici si possa trarre qualsivoglia informazione sulle leggi teoriche della fisica. Se ne concluderebbe pertanto che anche l'adozione stessa della cornice teorica quantistica, ad oggi motivata dal suo successo sperimentale, perderebbe il suo principale fondamento. In ultima analisi, la mancanza di potere predittivo rende tale teoria quantistica, mutilata del processo di misurazione, non falsificabile.

L'argomento precedente non solamente illustra come non si possa facilmente rimuovere dalla meccanica quantistica il dualismo tra sistema osservato e apparato di misura senza incorrere in gravi inconsistenze, ma suggerisce anche, come implicitamente indicato dalla teoria quantistica stessa, che la linea di demarcazione tra il sistema osservato e l'apparato sia di per sé mobile, non oggettiva, in altri termini relativa all'osservatore che descrive la misurazione.

Va detto per completezza che esistono varianti della meccanica quantistica in cui la linea di demarcazione è teorizzata come obiettiva, in particolare la teoria di Ghirardi, Rimini, e Weber (GRW) [5], e tutti 
i cosiddetti modelli di collasso spontaneo che ad essa si rifanno. In tal caso la demarcazione è codificata in opportune costanti spaziali e temporali che definiscono una scala al di sotto della quale si manifestano effetti quantistici, e al di sopra della quale invece i fenomeni sono esclusivamente classici. Le sfide che attualmente impegnano gli scienziati nello sviluppo di dispositivi quantistici di nuova generazione costituiscono indirettamente un tentativo di falsificare tale famiglia di teorie, o di porre limiti sempre più stretti ai valori delle loro costanti caratteristiche.

Se però ci atteniamo alla interpretazione della meccanica quantistica di Copenhagen, gli argomenti sopra illustrati portano a concludere che la scelta del modello matematico da adottare in un dato contesto abbia sempre una origine arbitraria e soggettiva. È forse necessario che l'adozione di tale visione scaturisca necessariamente in una posizione solipsistica? Sempre lo schema precedente ci permette di attenuare questa conclusione, sottolineando come le diverse descrizioni dell'esperimento date da diversi osservatori siano compatibili l'una con l'altra. Nonostante il carattere soggettivo di alcune scelte nell'attribuzione di una descrizione matematica agli enti fisici, la teoria permette una condivisione intersoggettiva consistente.

La scelta suddetta, quindi, è armonica con l'idea che la teoria fisica non descriva in modo autoevidente una realtà indipendente dall'osservazione, ma sia applicata mediante scelte arbitrarie dell'osservatore, che di volta in volta la utilizza per descrivere ogni specifico esperimento. In tal senso, la teoria fisica non consta di enti la cui semantica sia riferita direttamente all'ontologia dei sistemi fisici, ma risulti da una impresa intellettuale che contempla la partecipazione di diversi soggetti, insieme all'ambiente fisico, ed in cui i significati sono risultato di una negoziazione, ovvero del continuo confronto tra ipotesi e dati, che scaturisce nel costante aggiornamento delle ipotesi stesse. L'evoluzione dei significati gioca pertanto un ruolo fondante per l'interpretazione dei dati sensoriali in termini di ciò che conosciamo. In questo senso la conoscenza data dalla fisica ha un carattere assimilabile a quello attribuito alla conoscenza nella psicologia e pedagogia costruttivista, in particolare nella epistemologia genetica iniziata da Piaget [6], ambito nel quale il concetto di "negoziazione dei significati" fu introdotto.

Parimenti, lo status delle leggi fisiche quali algoritmi da seguire per trarre predizioni affidabili, piuttosto che come meccanismi insiti nella natura dell'universo, è paragonabile a quello dei valori all'etica 
costruttivista, in cui le leggi morali hanno carattere normativo, piuttosto che ontologico.

Quanto affermato sino a questo punto può essere messo in discussione se ci si pone la questione della completezza della teoria quantistica. È possibile immaginare che tale teoria non sia veramente fondamentale? Si può pensare l'indeterminatezza delle quantità fisiche tipica del mondo quantistico come pura misura di ignoranza? È chiaro infatti come la visione del ruolo gnoseologico della fisica sopra esposta dipenda dal valore che si dà alle strutture della meccanica quantistica, le quali hanno improntato tutta la riflessione sino a qui svolta. È naturalmente possibile che la fisica quantistica sia in futuro superata da nuove teorie. Ciò che vogliamo ora sostenere, tuttavia, è che tale osservazione non costituisca un indebolimento delle argomentazioni svolte in questa sede. Esse infatti, non richiedono nello specifico la teoria quantistica, ma sono estensibili a qualunque eventuale teoria post-quantistica, nella misura in cui questa contempli la non riducibilità del processo di misurazione ad una dinamica reversibile di sistemi chiusi.

Una teoria che eluda tale caratteristica deve necessariamente permettere la descrizione della misurazione come processo reversibile, che non introduca disturbo nell'evoluzione del sistema osservato, come rimarcato precedentemente nella discussione del principio di "no information without disturbance". Pertanto, in tale teoria, non ha spazio il concetto di complementarità.

La storia relativamente recente del teorema di Bell [7] e quella molto recente degli esperimenti $[8,9,10,11]$ di violazione delle disuguaglianze di Clauser-Horne-Shimony e Holt [12] ci hanno insegnato tuttavia che il prezzo epistemologico da pagare, per eliminare la complementarità, è quello di una realtà in cui il controllo locale sui sistemi diventa una illusione.

In estrema sintesi, infatti, la violazione della suddetta disuguaglianza, verificata sperimentalmente, implica che la produzione del risultato di una misura effettuata su di un sistema fisico non sia interpretabile come lo svelamento di una realtà che abbia le seguenti caratteristiche: i) indipendenza dalla scelta di grandezza da misurare e ii) indipendenza dai risultati di misure remote. Pertanto, o l'esistenza del risultato non è indipendente dalla scelta di misurare la grandezza in questione, oppure il valore della grandezza non dipende esclusivamente dal sistema che si osserva, ma in linea di principio dallo stato dell'intero universo. Ad esempio, per prevedere il valore dell'altezza del soffitto di 
una stanza, misurata ad un dato istante, è necessario considerare tutte le molecole di idrogeno che nella storia dell'universo abbiano interagito con quelle delle pareti della stanza. Chiaramente, molto difficilmente una tale teoria sarà predittiva.

L'interpretazione di Copenhagen della teoria quantistica prevede l'opzione filosoficamente più economica, ovvero quella per la prima alternativa: l'esistenza del risultato non è indipendente dalla scelta di misurare la grandezza in questione.

In conclusione, abbiamo discusso alcuni aspetti della teoria quantistica che riteniamo abbiano rilievo nella questione sulla natura della conoscenza scientifica. In particolare, l'attenzione è stata focalizzata sul legame tra la descrizione teorica dei sistemi e la loro fenomenologia, nel quale abbiamo individuato il principale oggetto del confronto tra le posizioni realiste e quella costruttivista che si vuole proporre. A tal proposito, si è osservato come le interpretazioni affini a quella cosiddetta di Copenhagen riescano a salvare il ruolo dei dati empirici, senza il quale le basi stesse della teoria quantistica sarebbero del tutto inconsistenti. Il ruolo centrale della misurazione in tali approcci riduce senz'altro l'ambizione di formulare una teoria che aderisca esattamente all'ontologia dei sistemi fisici. Tuttavia, la tesi che si vuole sostenere è che la principale conseguenza della rivoluzione quantistica sia proprio quella di minare alle fondamenta tale ambizione. Si individua pertanto nell'approccio costruttivista all'epistemologia quello che meglio è in grado di accogliere in seno la fisica moderna, la cui forza non è tanto quella di spiegare i fenomeni esattamente così come essi sono, quanto di sapersi evolvere, in una continua tensione verso il miglioramento del proprio potere predittivo.

\section{BIBLIOGRAFIA}

[1] Günther Ludwig. The Problem of Formulating an Axiomatics for Quantum Mechanics, pages 1-7. Springer Berlin Heidelberg, Berlin, Heidelberg, 1985.

[2] E. Casari. Questioni di filosofia della matematica. Filosofia della Scienza. Feltrinelli, 1976.

[3] Giacomo Mauro D'Ariano, Giulio Chiribella, and Paolo Perinotti. Quantum Theory from First Principles: An Informational Approach. Cambridge University Press, 2017.

[4] J. von Neumann and R.T. Beyer. Mathematical Foundations of Quantum Mechanics. Investigations in physics. Princeton University Press, 1955. 
[5] G.C. Ghirardi, A. Rimini, and T. Weber. A model for a unified quantum description of macroscopic and microscopic systems. In Luigi Accardi and Wilhelm von Waldenfels, editors, Quantum Probability and Applications II, pages 223-232, Berlin, Heidelberg, 1985. Springer Berlin Heidelberg.

[6] Jean Piaget. Psychologie et épistémologie. Denoël-Gonthier, Paris, 1970.

[7] J.S. Bell. On the einstein podolsky rosen paradox. Physics Physique Fizika, 1:195-200, Nov 1964.

[8] Alain Aspect, Philippe Grangier, and Gérard Roger. Experimental tests of realistic local theories via bell's theorem. Phys. Rev. Lett., 47:460-463, Aug 1981.

[9] Lynden K. Shalm, Evan Meyer-Scott, Bradley G. Christensen, Peter Bierhorst, Michael A. Wayne, Martin J. Stevens, Thomas Gerrits, Scott Glancy, Deny R. Hamel, Michael S. Allman, Kevin J. Coakley, Shellee D. Dyer, Carson Hodge, Adriana E. Lita, Varun B. Verma, Camilla Lambrocco, Edward Tortorici, Alan L. Migdall, Yanbao Zhang, Daniel R. Kumor, William H. Farr, Francesco Marsili, Matthew D. Shaw, Jeffrey A. Stern, Carlos Abellán, Waldimar Amaya, Valerio Pruneri, Thomas Jennewein, Morgan W. Mitchell, Paul G. Kwiat, Joshua C. Bienfang, Richard P. Mirin, Emanuel Knill, and Sae Woo Nam. Strong loophole-free test of local realism. Phys. Rev. Lett., 115:250402, Dec 2015.

[10] B. Hensen, H. Bernien, A.E. Dréau, A. Reiserer, N. Kalb, M.S. Blok, J. Ruitenberg, R.F.L. Vermeulen, R.N. Schouten, C. Abellán, W. Amaya, V. Pruneri, M.W. Mitchell, M. Markham, D.J. Twitchen, D. Elkouss, S. Wehner, T. H. Taminiau, and R. Hanson. Loophole-free bell inequality violation using electron spins separated by 1.3 kilometres. Nature, 526:682 EP -, 102015.

[11] Marissa Giustina, Marijn A. M. Versteegh, Sören Wengerowsky, Johannes Handsteiner, Armin Hochrainer, Kevin Phelan, Fabian Steinlechner, Johannes Kofler, Jan-Åke Larsson, Carlos Abellán, Waldimar Amaya, Valerio Pruneri, Morgan W. Mitchell, Jörn Beyer, Thomas Gerrits, Adriana E. Lita, Lynden K. Shalm, Sae Woo Nam, Thomas Scheidl, Rupert Ursin, Bernhard Wittmann, and Anton Zeilinger. Significant-loophole-free test of bell's theorem with entangled photons. Phys. Rev. Lett., 115:250401, Dec 2015.

[12] John F. Clauser, Michael A. Horne, Abner Shimonand Richard A. Holt. Proposed experiment to test local hidden-variable theories. Phys. Rev. Lett., 23:880-884, Oct 1969. 